\title{
Quest for localized 4D black holes in brane worlds
}

\author{
Panagiota Kanti \\ CERN, Theory Division, CH-1211 Geneva 23, Switzerland \\ and Scuola Normale Superiore, Piazza dei Cavalieri 7, I-56126 Pisa, Italy \\ Kyriakos Tamvakis \\ Physics Department, University of Ioannina, GR-451 10 Ioannina, Greece
}

(Received 3 November 2001; published 19 March 2002)

\begin{abstract}
We investigate the possibility of obtaining localized black hole solutions in brane worlds by introducing a dependence of the four-dimensional line element on the extra dimension. An analysis, performed for the cases of an empty bulk and of a bulk containing either a scalar or a gauge field, reveals that no conventional type of matter can support such a dependence. Considering a particular ansatz for the five-dimensional line element that corresponds to a black hole solution with a "decaying" horizon, we determine the bulk energy-momentum tensor capable of sustaining such behavior. It turns out that an exotic, shell-like distribution of matter is required. For such solutions, the black hole singularity is indeed localized near the brane and the spacetime is well defined near the AdS horizon, in contrast with the behavior found in black string type solutions.
\end{abstract}

DOI: 10.1103/PhysRevD.65.084010 PACS number(s): 04.50.+h, 04.70.Bw, 11.10.Kk, 11.25.Mj

\section{INTRODUCTION}

The recent studies on higher dimensional models of gravitation, motivated by the need to explain the large difference in magnitude between the Planck scale $M_{P} \sim 2 \times 10^{18} \mathrm{GeV}$ and the electroweak scale of particle physics, have resulted in a number of interesting theoretical ideas [1-4]. Among them, of particular interest, is the idea of an extra noncompact dimension [4]. The models proposed by Randall and Sundrum (RS) correspond to regions of AdS space separated by zero-thickness 3-branes. The standard model interactions are confined on such a 3-brane, while gravitation, propagating in the five-dimensional bulk, is represented on the brane by an ordinary massless graviton localized on it [5]. The four-dimensional Planck mass is an effective scale given in terms of a fundamental scale of five-dimensional gravity and the AdS radius of the five-dimensional spacetime.

The four-dimensional Schwarzschild metric describes the simplest case of gravitational collapse in the standard fourdimensional world. In a five-dimensional framework, as the Randall-Sundrum model, it would be natural to ask if matter trapped on the brane, when it undergoes gravitational collapse, could still be described by a Schwarzschild-type metric. The one-brane RS model has a factorized RSSchwarzschild black hole solution ${ }^{1}$ corresponding to a black string infinite in the fifth dimension [6]. The induced metric on the brane is purely Schwarzschild, respecting all the usual astrophysical constraints [7]. Although the Ricci scalar and the square of the Ricci tensor corresponding to this solution are everywhere finite, the square of the Riemann tensor diverges at the AdS horizon at infinity as

\footnotetext{
${ }^{1}$ The five-dimensional metric is $g_{\mu \nu}=e^{-2 \lambda|y|} g_{\mu \nu}^{(S)}, g_{55}=1$ with $g_{\mu \nu}^{(S)}=\operatorname{diag}\left(-1+2 M / r,(1-2 M / r)^{-1}, r^{2}, r^{2} \sin ^{2} \theta\right)$ being the fourdimensional Schwarzschild metric and $\lambda$ the five-dimensional AdS radius.
}

$$
R_{M N R S} R^{M N R S} \propto \frac{48 M^{2} e^{4 \lambda|y|}}{r^{6}} .
$$

This singularity renders the above solution physically unsuitable and has led to the speculation that there exists a localized black cigar solution which has a finite-extension along the fifth dimension due to a Gregory-Laflamme type of instability [8] near the AdS horizon. The subject of black holes in the context of extra dimensions has been extensively studied in recent years: black holes on branes [9], branes in a black hole background [10], black hole solutions emerging from the superstring or supergravity theory [11] and their stability [12], five- or higher-dimensional black hole solutions [13] and black holes in scenarios with large extra dimensions [14] are some of the topics studied so far.

The present article is devoted to a question that still remains open, namely to the existence of black hole solutions that reduce to the standard Schwarzschild black hole on the brane while having a finite extension along the fifth dimension. This amounts to finding solutions with a nonfactorizable $y$ dependence of the four-dimensional metric tensor $g_{\mu \nu}=e^{2 A(y)} \hat{g}_{\mu \nu}(x, y)$, with $\hat{g}_{\mu \nu}(x, 0)=g_{\mu \nu}^{(S)}(x)$, in contrast to the black string case where $g_{\mu \nu}=e^{2 A(y)} g_{\mu \nu}^{(S)}(x)$. The assumed $y$ dependence of the four-dimensional metric tensor would result in an additional scaling of the value of the black hole horizon with respect to the extra dimension, independently of the one of the warp factor. By choosing appropriately the $y$ dependence of $\hat{g}_{\mu \nu}(x, y)$, this could lead to the vanishing of the horizon away from the brane, but well before the AdS horizon, and thus to a possible localization of the black hole along the extra dimension. The choice of $\hat{g}_{\mu \nu}(x, y)$ should be consistent with the desired behavior of the metric tensor near or away from the brane as well as with the boundary (jump) conditions that the presence of the 3-brane introduces in the model.

Pursuing this idea, we first investigate the possibility that the desired dependence of the four-dimensional metric tensor 
on the extra dimension might be supported by an empty bulk or by a bulk containing either a scalar or a gauge field. The ansatz for the metric tensor is taken to be spherically symmetric, giving rise to $y$-dependent, neutral or charged, generalizations of the Schwarzschild and AdS/dS-Schwarzschild black hole solutions on the brane. Our analysis reveals that, for the chosen class of $y$ dependence, an empty bulk or a bulk dominated by a conventional form of matter cannot support such a $y$ dependence. We, then, reverse the question and ask what kind of bulk matter can give rise to such $y$-dependent solutions for the metric tensor. We consider a particular ansatz for the four-dimensional line element that is characterized by a rapidly "decaying" value of the black hole horizon away from the brane. The components of the corresponding bulk energy-momentum tensor are determined and their behavior, with respect to brane and bulk coordinates, is examined. It turns out that the matter distribution capable of sustaining localized black hole solutions is an exotic, shell-like one, localized around the brane and vanishing asymptotically at large distances from both the black hole horizon and the location of the brane. This particular solution is free from the singularity at the AdS horizon found in black string solutions and has the black hole singularity localized near the brane. However, it is accompanied by the appearance of a second, localized singularity at the black hole horizon due to the singular behavior of the bulk energymomentum tensor at the same point. We argue that this singularity is only due to the particular choice of the fivedimensional spacetime and that a more complicated choice, describing a four-dimensional black hole solution on the brane without possessing a horizon, might not have the same problem.

The paper has the following structure: in Sec. II we present the theoretical framework of our model, as well as the ansatz for the five-dimensional metric tensor, and derive the equations of motion. In Secs. III, IV, and V, we look for $y$-dependent solutions for the four-dimensional metric tensor in the case of an empty bulk and of a bulk containing either a scalar or a gauge field, respectively. Section VI contains the analysis of the alternative approach of demanding a localized black hole solution and examining the nature of the corresponding bulk matter. We present our conclusions in Sec. VII.

\section{EQUATIONS OF MOTION}

We shall consider the general framework defined by the five-dimensional Einstein action in the presence of a 3-brane located at an arbitrary point chosen to be $y=0$, namely,

$$
S=-\int d^{4} x d y \sqrt{-g}\left\{-\frac{R}{2 \kappa^{2}}+\Lambda_{B}+\frac{\sigma}{\sqrt{g_{55}}} \delta(y)-\mathcal{L}_{B}\right\},
$$

where $\kappa^{2}=8 \pi G_{5}$, with $G_{5}$ the five-dimensional Newton's constant. In the above, $\Lambda_{B}$ stands for a bulk cosmological constant, while any additional existing bulk matter is repre- sented by the Lagrangian $\mathcal{L}_{B}$. The constant $\sigma$ represents the positive brane tension. The general form of the metric will be taken to be

$$
d s^{2}=e^{2 A(y)} \hat{g}_{\mu \nu}(x, y) d x^{\mu} d x^{\nu}+d y^{2} .
$$

Throughout this article, we will be interested in solutions that are spherically symmetric on the brane, we therefore move to the ansatz

$$
\begin{aligned}
d s^{2}= & e^{2 A(y)}\left\{-U(r, y) d t^{2}+\hat{U}(r, y)^{-1} d r^{2}\right. \\
& \left.+r^{2}\left(d \theta^{2}+\sin ^{2} \theta d \varphi^{2}\right)\right\}+d y^{2} .
\end{aligned}
$$

In addition, we assume that the spacetime will remain invariant under a mirror transformation $y \rightarrow-y$. The case of the black string solution [6] is recovered for the RandallSundrum choice of the warp factor $A(y)=-\lambda|y|$, with $\lambda^{2}$ $=\kappa^{2}\left|\Lambda_{B}\right| / 6$, and

$$
\hat{U}(r, y)=U(r, y)=1-\frac{2 M}{r} .
$$

Another particular type of solutions that arises as a special case of the above metric ansatz is the RS-AdS/dSSchwarzschild one $[15,16,18,19]$ for

$$
e^{A(y)}=\cosh (\lambda y)-\frac{\kappa \sigma}{\sqrt{6\left|\Lambda_{B}\right|}} \sinh (\lambda|y|)
$$

and

$$
\hat{U}(r, y)=U(r, y)=1-\frac{2 M}{r}-\Lambda r^{2},
$$

where $\Lambda$ is the four-dimensional cosmological constant given by

$$
\Lambda=\frac{\kappa^{2}}{6}\left(\frac{\kappa^{2} \sigma^{2}}{6}-\left|\Lambda_{B}\right|\right) .
$$

Similarly to the RS-Schwarzschild solutions, the above solutions are characterized by finite $R$ and $R_{M N} R^{M N}$, while the square of the Riemann tensor is given by Eq. (1.1), where $e^{4 \lambda|y|}$ is replaced by $e^{-4 A(y)}$ according to Eq. (2.5). The de Sitter solution $(\Lambda>0)$ is characterized by a true singularity at a finite distance $y=y_{0}$ from the brane, with $y_{0}$ defined by

$$
\tanh \left(\lambda\left|y_{0}\right|\right)=\sqrt{\frac{6\left|\Lambda_{B}\right|}{\kappa^{2} \sigma^{2}}}
$$

thus causing the square of the Riemann tensor to diverge and exhibiting the same unnaturalness as the RS-Schwarzschild solution. For the anti-de Sitter solution $(\Lambda<0)$, all gravitational scalars are well defined everywhere; however, the divergence of the warp factor itself at infinite distance from the brane necessitates the introduction of a second brane for the 
recovery of the conventional four-dimensional gravity (for alternative methods, see Refs. $[17,18])$.

In the following analysis, we will be looking for $y$-dependent (neutral or charged) generalizations of the above solutions that reduce to conventional four-dimensional black hole solutions on the brane (Schwarzschild, ReissnerNordström or AdS/dS-Schwarzschild). In order to simplify the equations of motion resulting from the above action (2.1) and the metric ansatz (2.3), we will consider a restricted case, namely $U=\hat{U}$. Under this assumption, Einstein's equations of motion, written in terms of the Einstein tensor $G_{M N}=R_{M N}-\frac{1}{2} g_{M N} R$ and a bulk energy-momentum tensor, are

$$
\begin{aligned}
G_{t}^{t}= & 6 A^{\prime 2}+3 A^{\prime \prime}-\frac{2 A^{\prime} U^{\prime}}{U}+\frac{3 U^{\prime 2}}{4 U^{2}}-\frac{U^{\prime \prime}}{2 U}+\frac{U-1}{e^{2 A} r^{2}} \\
& +\frac{1}{e^{2 A} r} \frac{\partial U}{\partial r} \\
= & \kappa^{2}\left[-\Lambda_{B}-\sigma \delta(y)+T_{t}^{t}\right] \\
G_{r}^{r}= & 6 A^{\prime 2}+3 A^{\prime \prime}+\frac{2 A^{\prime} U^{\prime}}{U}-\frac{U^{\prime 2}}{4 U^{2}}+\frac{U^{\prime \prime}}{2 U}+\frac{U-1}{e^{2 A} r^{2}} \\
& +\frac{1}{e^{2 A} r} \frac{\partial U}{\partial r} \\
= & \kappa^{2}\left[-\Lambda_{B}-\sigma \delta(y)+T_{r}^{r}\right] \\
G_{\theta}^{\theta}= & 6 A^{\prime 2}+3 A^{\prime \prime}+\frac{U^{\prime 2}}{4 U^{2}}+\frac{1}{e^{2 A} r} \frac{\partial U}{\partial r}+\frac{1}{2 e^{2 A}} \frac{\partial^{2} U}{\partial r^{2}} \\
= & \kappa^{2}\left[-\Lambda_{B}-\sigma \delta(y)+T_{\theta}^{\theta}\right] \\
G_{\varphi}^{\varphi}= & G_{\theta}^{\theta}=\kappa^{2}\left[-\Lambda_{B}-\sigma \delta(y)+T_{\varphi}^{\varphi}\right] \\
G_{5}^{5}= & 6 A^{\prime 2}-\frac{U^{\prime 2}}{4 U^{2}}+\frac{U-1}{e^{2 A} r^{2}}+\frac{2}{e^{2 A} r} \frac{\partial U}{\partial r}+\frac{1}{2 e^{2 A}} \frac{\partial^{2} U}{\partial r^{2}} \\
= & \kappa^{2}\left[-\Lambda_{B}+T_{5}^{5}\right] \\
G_{r 5}= & -\frac{1}{U r} \frac{\partial U}{\partial y}-\frac{1}{2 U} \frac{\partial^{2} U}{\partial r \partial y}=\kappa^{2} T_{r 5},
\end{aligned}
$$

where a prime denotes differentiation with respect to $y$ and $T_{N}^{M}$ the bulk distribution of energy associated with the Lagrangian $\mathcal{L}_{B}$.

Taking the warp factor to be the Randall-Sundrum one, namely $e^{A(y)}=e^{-\lambda|y|}$, or the $\mathrm{RS}-\mathrm{AdS} / \mathrm{dS}-\mathrm{Schwarzschild}$ one (2.5), the equations of motion reduce to a set of partial differential equations for $U(r, y)$. In the former case, the terms involving solely derivatives of the function $A(y)$ cancel exactly the bulk cosmological constant term while in the latter case, we need to make the substitutions $T_{\nu}^{\mu} \rightarrow T_{\nu}^{\mu}$
$-3 \Lambda e^{-2 A}$ and $T_{5}^{5} \rightarrow T_{5}^{5}-6 \Lambda e^{-2 A}$. The components of Einstein's equations containing second derivatives with respect to the extra coordinate will give rise to the jump conditions at the location of the brane. For the RS-Schwarzschild solution, the fine-tuning $\lambda=\kappa^{2} \sigma / 6$ is still required, while for the RSAdS/dS-Schwarzschild one it is the location of the minimum or singularity, and thus the size of the extra dimension, that is fixed in terms of the fundamental parameters of the theory. In both cases, however, the same equations demand the continuity of the metric function $U(r, y)$ along the extra dimension, a constraint that will prove to be of great importance in the following analysis.

We shall now consider and analyze the equations for $U(r, y)$ in various cases, namely, in the case of empty bulk and in the cases of bulk containing either a scalar or a gauge field.

\section{EMPTY BULK}

In this case, all the components of $T_{N}^{M}$ are assumed to be zero. The off-diagonal component (2.14) can then be easily integrated to give the solution

$$
U(r, y)=\frac{\lambda(y)}{r^{2}}+f(r)
$$

where $\lambda(y)$ and $f(r)$ are arbitrary functions. We impose the condition that, in the limit $y \rightarrow 0, \lambda(y)$ is a well-defined function. Moreover, in order to ensure the correct asymptotic behavior at infinity for each AdS slice, we rewrite the above solution as

$$
U(r, y)=1+\frac{\lambda(y)}{r^{2}}+\sum_{n} \alpha_{n} r^{n}
$$

where $n$ is an integer number (strictly negative for the Schwarzschild and Reissner-Nordstrom black hole solutions on the brane, both negative and positive for the AdS/dSSchwarzschild ones) and $\alpha_{n}$ arbitrary constants. Taking the sum of Eqs. (2.11) and (2.13), we find the constraint ${ }^{2}$

$$
\frac{U-1}{e^{2 A} r^{2}}+\frac{3}{e^{2 A} r} \frac{\partial U}{\partial r}+\frac{1}{e^{2 A}} \frac{\partial^{2} U}{\partial r^{2}}=\left\{\begin{array}{c}
-9 \Lambda e^{-2 A} \\
0
\end{array}\right\} .
$$

Upon substituting the solution (3.2) in the above expression, it reduces to

$$
\frac{\lambda(y)}{r^{4}}+\sum_{n} \alpha_{n} r^{n-2}(n+1)^{2}=\left\{\begin{array}{c}
-9 \Lambda \\
0
\end{array}\right\}
$$

with the unique solution

\footnotetext{
${ }^{2}$ Whenever necessary, the upper entry of a two-line matrix will stand for the result for a "bent" 3-brane $(\Lambda \neq 0)$ while the lower entry will stand for a flat 3-brane $(\Lambda=0)$.
} 


$$
\begin{gathered}
\lambda(y)=0 \\
\left\{\begin{array}{c}
n=-1, a_{-1}=\text { const. } \quad \text { and } n=2, a_{2}=-\Lambda \\
n=-1, a_{-1}=\text { const. }
\end{array}\right\},
\end{gathered}
$$

which is nothing else than the RS-AdS/dS-Schwarzschild and the black string RS-Schwarzschild solution, respectively, for $a_{-1}=-2 M$. We therefore conclude that, for the assumed asymptotic behavior, any $y$ dependence, described by the ansatz (2.3) with $\hat{g}_{t t}=-1 / \hat{g}_{r r}$, is ruled out in the case of an empty bulk (see also [20]) and a non-trivial energy distribution is required for this purpose.

\section{A BULK SCALAR FIELD}

In the presence of a five-dimensional scalar field in the bulk, the components of $T_{N}^{M}$ assume the form

$$
\begin{aligned}
T_{t}^{t} & =-\frac{U}{2} e^{-2 A}\left(\partial_{r} \phi\right)^{2}-\frac{\phi^{\prime 2}}{2}-V(\phi), \\
T_{r}^{r} & =\frac{U}{2} e^{-2 A}\left(\partial_{r} \phi\right)^{2}-\frac{\phi^{\prime 2}}{2}-V(\phi), \\
T_{\theta}^{\theta} & =T_{\varphi}^{\varphi}=T_{t}^{t}, \\
T_{5}^{5} & =-\frac{U}{2} e^{-2 A}\left(\partial_{r} \phi\right)^{2}+\frac{\phi^{\prime 2}}{2}-V(\phi), \\
T_{r 5} & =\left(\partial_{r} \phi\right) \phi^{\prime} .
\end{aligned}
$$

Let us first assume that the bulk scalar field depends only on the extra coordinate $y$. Then, the off-diagonal component of $T_{N}^{M}$ is again zero, which leads once again to the solution (3.2) for the metric function $U(r, y)$. Taking again the sum of Eqs. (2.11) and (2.13), we now find

$$
\begin{array}{r}
\frac{U-1}{e^{2 A} r^{2}}+\frac{3}{e^{2 A} r} \frac{\partial U}{\partial r}+\frac{1}{e^{2 A}} \frac{\partial^{2} U}{\partial r^{2}} \\
=-2 \kappa^{2} V(\phi)+\left\{\begin{array}{c}
-9 \Lambda e^{-2 A} \\
0
\end{array}\right\}
\end{array}
$$

or

$$
\frac{\lambda(y)}{r^{4}}+\sum_{n} \alpha_{n} r^{n-2}(n+1)^{2}=-2 \kappa^{2} V(\phi) e^{2 A}+\left\{\begin{array}{c}
-9 \Lambda \\
0
\end{array}\right\} .
$$

The right-hand side (rhs) of the above equation is a pure function of $y$ while the left-hand side is not. Therefore, the only solution is

$$
\lambda(y)=V(\phi)=0
$$

$$
\left\{\begin{array}{c}
n=-1, a_{-1}=\text { const. } \quad \text { and } \quad n=2, a_{2}=-\Lambda \\
n=-1, a_{-1}=\text { const. }
\end{array}\right\},
$$

which reduces again to the $\mathrm{RS}-\mathrm{AdS} / \mathrm{dS}-\mathrm{Sch}$ warzschild and RS-Schwarzschild solutions, respectively, excluding any $y$ dependence of the 4D metric function.

The solution (3.2) for $U(r, y)$ still holds if we assume instead that the bulk scalar field depends only on the brane coordinate $r$. In that case, we proceed as follows. Rearranging appropriately the pairs of Eqs. (2.9), (2.10) and (2.11), (2.13), we find the constraints

$$
\begin{gathered}
\frac{2(U-1)}{e^{2 A} r^{2}}+\frac{2}{e^{2 A} r} \frac{\partial U}{\partial r}+\frac{U^{\prime 2}}{2 U^{2}}=-2 \kappa^{2} V(\phi)+\left\{\begin{array}{c}
-6 \Lambda e^{-2 A} \\
0
\end{array}\right\}, \\
\frac{U^{\prime 2}}{2 U^{2}}=\frac{U-1}{e^{2 A} r^{2}}+\frac{1}{e^{2 A} r} \frac{\partial U}{\partial r}+\left\{\begin{array}{c}
3 \Lambda e^{-2 A} \\
0
\end{array}\right\}
\end{gathered}
$$

respectively. Subtracting the above two constraints, we find

$$
\frac{U-1}{r^{2}}+\frac{1}{r} \frac{\partial U}{\partial r}=-\frac{2}{3} e^{2 A} \kappa^{2} V(\phi)+\left\{\begin{array}{c}
-3 \Lambda \\
0
\end{array}\right\}
$$

which leads to

$$
-\frac{\lambda(y)}{r^{4}}-\sum_{n} \alpha_{n} r^{n-2}(n+1)=-\frac{2}{3} e^{2 A} \kappa^{2} V(\phi)+\left\{\begin{array}{c}
-3 \Lambda \\
0
\end{array}\right\} .
$$

In this case, the rhs of the above equation is a product of the discontinuous warp factor times a function of $r$. Since, according to the jump conditions, the left-hand side (lhs) is continuous in $y$, this equation cannot be maintained with a non-trivial potential and a non-constant $\lambda$. Thus, in this case also we have only the black string solution and its AdS/dS generalization (2.5), (2.6).

Next, we consider the more general case where the bulk scalar field depends on both coordinates. The rearrangement of Eqs. (2.9), (2.10) and (2.11), (2.13) leads again, in the same way as above, to the constraint (4.7). The off-diagonal component of Einstein's equations (2.14) cannot be integrated now due to the presence of the non-vanishing component $T_{r 5}$. However, motivated by the solution for $U$ derived in earlier cases, we assume the form:

$$
U(r, y)=1-\sum_{n} \alpha_{n}(y) r^{n}
$$

where $n$ is again an integer number. Taking the sum of Eqs. (2.11) and (2.13) and using the constraint (4.6), we obtain

$$
\begin{aligned}
\kappa^{2} U\left(\partial_{r} \phi\right)^{2} & =\frac{2(U-1)}{r^{2}}-\frac{\partial^{2} U}{\partial r^{2}} \\
& =\sum_{n} \alpha_{n}(y) r^{n-2}(n+1)(n-2),
\end{aligned}
$$


which is indeed positive for $\alpha_{n}>0$ and $n<-1$ and/or $n$ $>2$. Note that, in agreement with the conclusion drawn in the case of an empty bulk, in the absence of a bulk scalar field, only the terms $n=-1$ and $n=2$, the mass term and the cosmological constant term, survive in the expression of the 4D metric function. Subtracting finally Eqs. (2.9) and (2.10), we find

$$
\frac{4 A^{\prime} U^{\prime}}{U}-\frac{U^{\prime 2}}{U^{2}}+\frac{U^{\prime \prime}}{U}=\kappa^{2} U e^{-2 A}\left(\partial_{r} \phi\right)^{2}
$$

or, using Eq. (4.9),

$$
\begin{gathered}
-\left(1-\sum_{m} \alpha_{m} r^{m}\right) \sum_{n} r^{n}\left(4 A^{\prime} \alpha_{n}^{\prime}+\alpha_{n}^{\prime \prime}\right)-\sum_{n, m} \alpha_{n}^{\prime} \alpha_{m}^{\prime} r^{n+m} \\
=e^{-2 A} \sum_{n} \alpha_{n} r^{n-2}(n+1)(n-2)\left(1-\sum_{m} \alpha_{m} r^{m}\right)^{2} .
\end{gathered}
$$

Collecting terms of order $r^{n}$, we are forced to impose the condition $4 A^{\prime} \alpha_{n}^{\prime}+\alpha_{n}^{\prime \prime}=0$, which, apart from being contradictory to our assumptions for continuous $\alpha_{n}(y)$, it is also disastrous in two ways: first, it leads to the result $\alpha_{n}(y)$ $\sim e^{-4 A}$, which has a behavior opposite to that of the warp factor-namely, as gravity becomes weaker, the "charges" at infinity become larger; secondly, in that case, what is left from the above equation can be rewritten as

$$
\kappa^{2} U e^{-2 A}\left(\partial_{r} \phi\right)^{2}=-\frac{U^{\prime 2}}{U^{2}}
$$

which is inconsistent given the reality of the metric function and of the bulk scalar field.

\section{A BULK GAUGE FIELD}

Next, we consider the case where, in addition to the bulk cosmological constant, a bulk gauge field gives rise to a nonconstant energy-momentum tensor

$$
T_{M N}=F_{M P} F_{N}^{P}-\frac{1}{4} g_{M N} F_{A B} F^{A B} .
$$

We start our analysis with some simple, but characteristic, choices for the gauge field configuration. We first assume that the only non-vanishing component of the gauge field is $A_{0}=a(y)$, inspired by the choice made by Visser in Ref. [21]. For this particular choice, we obtain the following components of the bulk energy-momentum tensor:

$$
T_{t}^{t}=T_{5}^{5}=-\frac{e^{-2 A} a^{\prime 2}}{2 U}, \quad T_{r}^{r}=T_{\theta}^{\theta}=T_{\varphi}^{\varphi}=\frac{e^{-2 A} a^{\prime 2}}{2 U} .
$$

Since the field strength has only one non-vanishing component, $F_{t 5}$, the off-diagonal energy-momentum tensor component $T_{r 5}$ is identically zero. The integration of Eq. (2.14), then, leads again to the solution (3.2) for the metric function $U(r, y)$. Despite the presence of a bulk $T_{M N}$, the analysis here closely follows the one performed in the case of an empty bulk. Since $T_{\theta}^{\theta}$ and $T_{5}^{5}$ are equal but of opposite sign, the sum of Eqs. (2.11) and (2.13) leads to the constraint (3.3) and thus to the unique RS-AdS/dS-Schwarzschild and RSSchwarzschild solutions.

An alternative choice could be the one in which the sole component of the gauge field is $r$ dependent instead of $y$ dependent: $A_{0}=a(r)$. What we actually do, in this case, is that we consider a usual four-dimensional ansatz for the gauge field configuration, that of a pure electric field, embedded in an extra dimension. Then, the components of the bulk energy-momentum tensor assume the form

$$
T_{t}^{t}=T_{r}^{r}=-\frac{e^{-4 A}}{2}\left(\partial_{r} a\right)^{2}, \quad T_{\theta}^{\theta}=T_{\varphi}^{\varphi}=T_{5}^{5}=\frac{e^{-4 A}}{2}\left(\partial_{r} a\right)^{2} .
$$

The off-diagonal component of Einstein's equations can be integrated once again, leading to the same solution (3.2) for $U(r, y)$. Turning next to the diagonal components, we consider the sum of Eqs. (2.11) and (2.13), which reduces to

$$
\begin{aligned}
& \frac{U-1}{e^{2 A} r^{2}}+\frac{3}{e^{2 A} r} \frac{\partial U}{\partial r}+\frac{1}{e^{2 A}} \frac{\partial^{2} U}{\partial r^{2}} \\
= & \kappa^{2} e^{-4 A}\left(\partial_{r} a\right)^{2}+\left\{\begin{array}{c}
-9 \Lambda e^{-2 A} \\
0
\end{array}\right\},
\end{aligned}
$$

or, alternatively,

$$
\frac{\lambda(y)}{r^{4}}+\sum_{n} \alpha_{n} r^{n-2}(n+1)^{2}=\kappa^{2} e^{-2 A}\left(\partial_{r} a\right)^{2}+\left\{\begin{array}{c}
-9 \Lambda \\
0
\end{array}\right\} .
$$

The above constraint is satisfied by the solution

$$
\begin{aligned}
\lambda(y)= & \lambda_{0} e^{-2 A(y)}, \quad\left(\partial_{r} a\right)^{2}=\frac{\lambda_{0}}{\kappa^{2} r^{4}}, \\
& \left\{\begin{array}{c}
n=-1, a_{-1}=\text { const. } \quad \text { and } \quad n=2, a_{2}=-\Lambda \\
n=-1, a_{-1}=\text { const. }
\end{array}\right\},
\end{aligned}
$$

which is not admissible because of the discontinuous $\lambda$. It is interesting to note that this "would-be" solution restores the "mass" term $n=-1$ in the expression of the metric function, and/or the cosmological constant term $n=2$, and leads to the conventional expression for the "electric" gauge field, i.e., $a(r)=Q_{e} / r$, with $Q_{e}= \pm \sqrt{\lambda_{0}} / \kappa$. However, the rearrangement of Eqs. (2.9), (2.10) and (2.11), (2.13) leads to constraints similar to Eq. (4.6), the only difference being the replacement of $2 V(\phi)$ by $e^{-4 A}\left(\partial_{r} a\right)^{2}$. The combination of these two constraints can be rewritten as 


$$
\frac{3 U^{\prime 2}}{2 U^{2}}=-\kappa^{2} e^{-4 A}\left(\partial_{r} a\right)^{2}<0 .
$$

It is impossible to satisfy this constraint and, therefore, we are forced to exclude the specific gauge field configuration used in the above analysis.

We will now address the most general case of having a five-dimensional gauge field that depends both on bulk and brane coordinates. In order to write down a sufficiently general ansatz for the gauge field, we turn for an instant to the four-dimensional case and to the most general black hole solution, i.e., the Kerr-Newman solution. The gauge field configuration that gives rise to such a solution has the form [22]

$$
\begin{aligned}
A_{\mu} d x^{\mu}= & \frac{Q_{e} r}{\rho^{2}}\left[d t-A_{J} \sin ^{2} \theta d \varphi\right] \\
& +\frac{Q_{m} \cos \theta}{\rho^{2}}\left[A_{J} d t-\left(r^{2}+A_{J}^{2}\right) d \varphi\right],
\end{aligned}
$$

where $A_{J}$ is the angular momentum and $\rho^{2}=r^{2}+A_{J}^{2} \cos ^{2} \theta$. Since here we are interested in black hole solutions that are characterized by a spherical symmetry, we may set $A_{J}=0$, which reduces the black hole solution to the ReissnerNordstrom one. Note that the presence of the radial magnetic field does not change at all the form of the solution for the metric tensor: it merely replaces the $Q_{e}^{2}$ metric parameter by $Q^{2}=Q_{e}^{2}+Q_{m}^{2}$. Therefore, for simplicity, we may ignore the $\theta$ dependence of the $A_{3}$ component of the gauge field that gives rise to the radial magnetic field. Coming back to the $5 \mathrm{D}$ case and generalizing the form of the gauge field, we may write the following ansatz:

$$
\begin{gathered}
A_{0}=A_{0}(r, y), \quad A_{1}=A_{1}(y), \quad A_{2}=A_{2}(y), \\
A_{3}=A_{3}(y), \quad A_{5}=A_{5}(r, y) .
\end{gathered}
$$

Another simplification is in order: the spherical symmetry, which characterizes our ansatz for the metric tensor (2.3), leads to the relation $G_{\theta}^{\theta}=G_{\varphi}^{\varphi}$ for the Einstein tensor, which subsequently demands the same relation for the corresponding components of the bulk energy-momentum tensor. It is straightforward to see that this leads to the constraint $A_{2}$ $=A_{3}=0$. That leaves us with the following expressions for the components of $T_{N}^{M}$ :

$$
\begin{aligned}
& T_{t}^{t}=F_{t r} F^{t r}+F_{t 5} F^{t 5}-\frac{1}{4} F^{2}, \\
& T_{r}^{r}=F_{t r} F^{t r}+F_{r 5} F^{r 5}-\frac{1}{4} F^{2},
\end{aligned}
$$

$$
T_{\theta}^{\theta}=T_{\varphi}^{\varphi}=-\frac{1}{4} F^{2},
$$

$$
\begin{gathered}
T_{5}^{5}=F_{t 5} F^{t 5}+F_{r 5} F^{r 5}-\frac{1}{4} F^{2} \\
T_{r 5}=g^{t t} F_{t r} F_{t 5},
\end{gathered}
$$

where

$$
F^{2}=F_{A B} F^{A B}=2\left(F_{t r} F^{t r}+F_{t 5} F^{t 5}+F_{r 5} F^{r 5}\right) .
$$

We first turn to the off-diagonal component (2.14): if we use again the generalized ansatz (4.9), this can be rewritten as

$$
\begin{aligned}
\frac{\partial\left(r^{2} U^{\prime}\right)}{\partial r} & =-\sum_{n} \alpha_{n}^{\prime}(y)(n+2) r^{n+1} \\
& =2 \kappa^{2} e^{-2 A}\left(\partial_{r} A_{0}\right) A_{0}^{\prime}
\end{aligned}
$$

According to the above, and in agreement with the conclusions drawn in the first two cases considered, when the $A_{0}$ component of the gauge field depends only on one of the two coordinates, the only $y$-dependent term that survives in the series of Eq. (4.8) is the $n=-2$ term, and even this one turns out to be trivial according to the aforementioned arguments. It is only in the case where this component has a non-trivial dependence on both coordinates that other $y$-dependent terms, including a "mass" term $(n=-1)$, might arise. Note that this is qualitatively different from what happens in four dimensions: there, an "empty" spacetime around a spherical body gives rise to a term proportional to $1 / r$, while the introduction of a gauge field leads to a higher term of $\mathcal{O}\left(1 / r^{2}\right)$.

We now consider the sum of the pairs of Eqs. (2.9), (2.10) and (2.11), (2.13). They have the form

$$
\begin{aligned}
& \frac{2(U-1)}{e^{2 A} r^{2}}+\frac{2}{e^{2 A} r} \frac{\partial U}{\partial r}+\frac{U^{\prime 2}}{2 U^{2}} \\
& =-\kappa^{2} e^{-4 A}\left(\partial_{r} A_{0}\right)^{2}+\left\{\begin{array}{c}
-6 \Lambda e^{-2 A} \\
0
\end{array}\right\}, \\
& \frac{U-1}{e^{2 A} r^{2}}+\frac{3}{e^{2 A} r} \frac{\partial U}{\partial r}+\frac{1}{e^{2 A}} \frac{\partial^{2} U}{\partial r^{2}} \\
& =\kappa^{2} e^{-4 A}\left(\partial_{r} A_{0}\right)^{2}+\left\{\begin{array}{c}
-9 \Lambda e^{-2 A} \\
0
\end{array}\right\},
\end{aligned}
$$

respectively. Taking the sum of the above two constraints, Eqs. (5.13) and (5.14), we arrive at the equation 


$$
\begin{gathered}
\frac{3(U-1)}{e^{2 A} r^{2}}+\frac{5}{e^{2 A} r} \frac{\partial U}{\partial r}+\frac{1 \partial^{2} U}{e^{2 A} \partial r^{2}} \\
=-\frac{U^{\prime 2}}{2 U^{2}}+\left\{\begin{array}{c}
-15 \Lambda e^{-2 A} \\
0
\end{array}\right\}
\end{gathered}
$$

or, alternatively, at

$$
\begin{aligned}
& \left(\sum_{n} \alpha_{n}(y)(n+1)(n+3) r^{n-2}-\left\{\begin{array}{c}
15 \Lambda \\
0
\end{array}\right\}\right) \\
& \quad \times\left[1-\sum_{m} \alpha_{m}(y) r^{m}\right]^{2}=\frac{e^{2 A}}{2} \sum_{n, m} \alpha_{n}^{\prime}(y) \alpha_{m}^{\prime}(y) r^{n+m} .
\end{aligned}
$$

Collecting terms of the same order in the above expression, we may easily see that severe constraints arise for the coefficients $\alpha_{n}$ and their derivatives. For example, in the case where $\Lambda=0$, considering terms up to $n=-3$ in the above series, we find that we always have $\alpha_{-1}^{\prime}=0$ and either $\alpha_{-2}^{\prime}$ $=\alpha_{-3}^{\prime}=0$ or even $\alpha_{-2}=\alpha_{-3}=0$. In either case, it is clear that the assumed dependence on the bulk coordinate of the 4D metric function $U$ is forbidden. For $\Lambda \neq 0$, and for terms up to $|n|=2$, we arrive at the unique solution $\alpha_{-1}^{\prime}=0$, $\alpha_{-2}=\alpha_{1}=0$ and $\alpha_{2}=\Lambda$, which is nothing else than the $y$-independent AdS/dS-Schwarzschild metric function (2.6). We may therefore conclude that even in the presence of a bulk gauge field, which depends on both brane and bulk coordinates, black hole solutions of the form (2.3), with the 4D metric function given by the generalized expression (4.8), are not allowed.

\section{AN ALTERNATIVE APPROACH}

From the results drawn in the previous section, it became clear that in the case of an empty bulk, apart from the contribution coming from the five-dimensional cosmological constant, and/or in the presence of conventional bulk matter in the form of either a five-dimensional scalar or gauge field, black hole solutions that respect the localization of gravity à la Randall-Sundrum and, at the same time, can accommodate a non-trivial $y$ dependence of the four-dimensional metric function cannot exist. The question that arises next is this: if solutions with $y$ dependence do exist, such that the value of the horizon itself scales with the extra dimension, reaching a zero value before the AdS horizon is reached, what is the nature of the bulk matter that can support these solutions? And, moreover, what is the distribution of bulk matter along the extra dimension? Does it mimic the localization of graviton zero mode and black hole horizon and have its peak near the 3-brane, or does it spread all the way up to the AdS horizon?

In order to answer the above questions, we will follow an alternative approach. We will demand that the 4D metric function $U$ has indeed a non-trivial dependence on the bulk coordinate, which is consistent with the desired behavior at small and large distances from the brane, and we will subse- quently determine the components of the unknown bulk energy-momentum tensor. Their profile in both the bulk and brane coordinates will be checked and an approximate equation of state, as well as some characteristic features of the bulk matter distribution, will be determined.

To this end, we consider the following ansatz for the line element of the five-dimensional spacetime that serves the above purpose:

$$
\begin{aligned}
d s^{2}= & e^{2 A(y)}\left\{-\left(1-\frac{w(y)}{r}\right) d t^{2}+\left(1-\frac{w(y)}{r}\right)^{-1} d r^{2}\right. \\
& \left.+r^{2}\left(d \theta^{2}+\sin ^{2} \theta d \varphi^{2}\right)\right\}+d y^{2}
\end{aligned}
$$

The above ansatz corresponds to a generalized $y$-dependent form of the RS-Schwarzschild solution (2.4) in which the value of the horizon of the four-dimensional black hole scales with the extra coordinate. We will also assume that the conformal factor that multiplies the 4D line element has the same profile as in the RS model, $e^{A(y)}=e^{-\lambda|y|}$. The arbitrary function $w(y)$ will be chosen to have the following behavior: $\lim _{y \rightarrow 0} w(y) \rightarrow r_{h}$ and $\lim _{y \rightarrow \infty} w(y) \rightarrow 0$. In this way, we ensure that the induced metric on the brane is of the Schwarzschild type and that the value of the black hole horizon vanishes before the AdS horizon is reached.

For the above spacetime background (6.1), Einstein's equations in the bulk take the form

$$
\begin{gathered}
3\left(A^{\prime \prime}+2 A^{\prime 2}\right)+\frac{2 A^{\prime} w^{\prime}}{(r-w)}+\frac{w^{\prime \prime}}{2(r-w)}+\frac{3 w^{\prime 2}}{4(r-w)^{2}} \\
=\kappa^{2}\left[-\Lambda_{B}+T_{t}^{t}\right] \\
3\left(A^{\prime \prime}+2 A^{\prime 2}\right)-\frac{2 A^{\prime} w^{\prime}}{(r-w)}-\frac{w^{\prime \prime}}{2(r-w)}-\frac{w^{\prime 2}}{4(r-w)^{2}} \\
=\kappa^{2}\left[-\Lambda_{B}+T_{r}^{r}\right] \\
3\left(A^{\prime \prime}+2 A^{\prime 2}\right)+\frac{w^{\prime 2}}{4(r-w)^{2}}=\kappa^{2}\left[-\Lambda_{B}+T_{\theta}^{\theta}\right] \\
6 A^{\prime 2}-\frac{w^{\prime 2}}{4(r-w)^{2}}=\kappa^{2}\left[-\Lambda_{B}+T_{5}^{5}\right] \\
\frac{w^{\prime}}{2 r(r-w)}=\kappa^{2} T_{r 5} .
\end{gathered}
$$

If we adopt the RS choice for the warp factor, the terms proportional to $A^{\prime 2}$ and $A^{\prime \prime}$ cancel exactly the bulk cosmological constant and the brane term, leaving the remaining terms involving derivatives of the unknown function $w(y)$ to determine the components of the bulk energy-momentum tensor $T_{N}^{M}$. It is straightforward to check that the resulting components trivially satisfy the equation for the conservation 
of energy and momentum, $D_{M} T_{N}^{M}=0$, for arbitrary $w$. Given the fact that the ansatz for the four-dimensional metric function $U(r, y)$ considered above is a special case of the more general ansatz (4.9), it becomes clear that the above nonconstant, bulk matter distribution can never be attributed to either a scalar or a gauge 5D field minimally coupled to gravity.

In order to investigate the properties of the aforementioned bulk matter distribution, we will check its profile along both brane $(r)$ and bulk $(y)$ coordinates. For this reason, we need to specify the metric function $w(y)$, which apparently determines both the profile of the black hole horizon along the extra dimension and the distribution of energy in the bulk. A choice that respects the asymptotic conditions imposed on the $w$ function both near and away from the brane is $w(y)=r_{h} e^{-a y^{2}}$, where $r_{h}$ is the fourdimensional value of the black hole horizon and $a$ an arbitrary, positive constant. In this case, in the limit $y \rightarrow 0$, we recover the Schwarzschild metric of a black hole located on the brane, as demanded. On the other hand, in the limit $y$ $\rightarrow \pm \infty$, i.e., at large distances from the brane, the horizon of the black hole shrinks to zero. Moreover, this particular choice is consistent with the demand that the fourdimensional metric function $U(r, y)$ be continuous along the extra dimension, since $\left.\left[U^{\prime}\right]_{y=0} \sim y e^{-a y^{2}}\right|_{y=0}=0$, and the physical demand that the bulk matter distribution decay faster than the AdS horizon.

For the aforementioned choice of the $w$ function, the components of the bulk $T_{N}^{M}$ come out to be

$$
\begin{gathered}
\kappa^{2} T_{t}^{t}=\frac{r_{h}\left(4 \lambda|y|+2 a y^{2}-1\right) a e^{-a y^{2}}}{(r-w)}+\frac{3 a^{2} y^{2} r_{h}^{2} e^{-2 a y^{2}}}{(r-w)^{2}}, \\
\kappa^{2} T_{5}^{5}=-\kappa^{2} T_{\theta}^{\theta}=\frac{\kappa^{2}}{2}\left(T_{t}^{t}+T_{r}^{r}\right), \\
\kappa^{2} T_{r}^{r}=-\frac{r_{h}\left(4 \lambda|y|+2 a y^{2}-1\right) a e^{-a y^{2}}}{(r-w)}-\frac{a^{2} y^{2} r_{h}^{2} e^{-2 a y^{2}}}{(r-w)^{2}}, \\
\kappa^{2} T_{r 5}=-\frac{a y r_{h} e^{-a y^{2}}}{r(r-w)} .
\end{gathered}
$$

The behavior of the three independent components of the energy-momentum tensor in the bulk, $T_{t}^{t}, T_{r}^{r}$ and $T_{r 5}$, with respect to the $(r, y)$ coordinates, is depicted in Fig. 1. The figure shows an example of a distribution of bulk matter capable of sustaining a localized black hole solution. In terms of the $r$ coordinate, and for each AdS slice $y=$ const, all the energy-momentum tensor components decrease as the distance from the black hole horizon increases, and eventually vanish as we approach the asymptotically flat regime $r$ $\rightarrow \infty$. Near the black hole horizon all components diverge, the diagonal ones faster, the off-diagonal slower. The singularity of the energy-momentum tensor at the horizon is a reflection of an analogous singularity of the scalar curvature

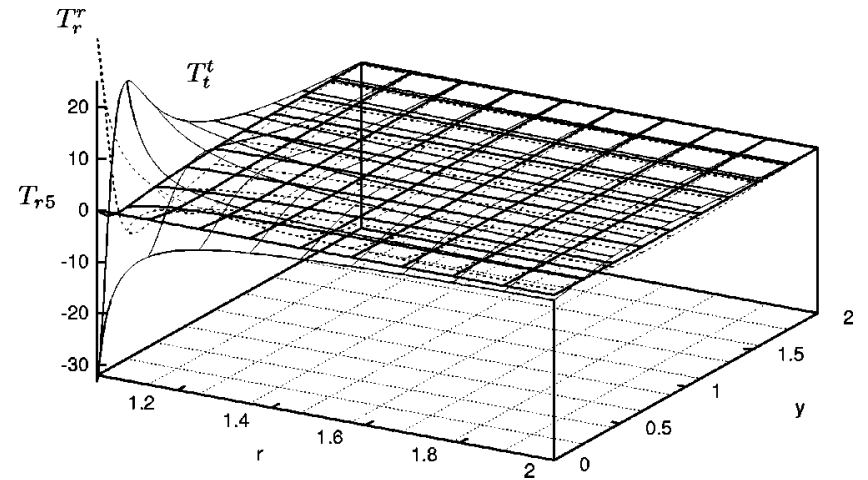

FIG. 1. The plot depicts the profile of the three components of the bulk energy-momentum tensor, $T_{t}^{t}, T_{r}^{r}$ and $T_{r 5}$, along the radial ( $r$ ) and bulk $(y)$ coordinate, for $\lambda=a=r_{h}=1$ and $w(y)=r_{h} e^{-a y^{2}}$. Both profiles "decay" very fast ensuring that all components vanish at large distance from the brane and/or the horizon of the black hole.

$R \sim w^{\prime 2} /(r-w)^{2}$. As we will shortly see, a more complicated choice of metric might not have this problem. Since the singularity at the horizon does not modify the issues of interest, namely the localized nature of solutions and the curvature singularity structure at infinity, we chose to stick with this simple choice and proceed.

Turning to the $y$ profile of the bulk matter and keeping $r$ fixed, we may easily see that the distribution of matter is localized near, but off, the 3-brane. All components rapidly decrease as one moves away from the brane, acquiring vanishing values as $y \rightarrow \infty$, while, at the location of the brane, all components are finite. The profile along the extra coordinate becomes clearer in Fig. 2: the components $T_{t}^{t}$ and $T_{r}^{r}$ are the only ones that have non-vanishing values at the location of the brane, while all the remaining ones vanish. The distribution of bulk matter is symmetric with respect to the brane. All components reach their peak in absolute values, slightly off, and on both sides of, the brane, thus creating a symmetric "shell" that engulfs the brane.

If we define a bulk energy density and pressure in the following way: $\rho=-T_{t}^{t}, p_{i}=T_{i}^{i}, p_{5}=T_{5}^{5}$ and $p_{r 5}=T_{r 5}$, we may notice some more features, in the distribution of bulk matter, emerging from Fig. 2. First, the dominant components $T_{t}^{t}$ and $T_{r}^{r}$ behave, throughout the extra dimension, in such a way that they always satisfy a stiff equation of state, i.e., $\rho=p_{r}$. All the remaining components acquire a relatively small maximum value slightly off the brane while they decay very fast as one moves close to or away from the brane. Coming back to the dominant components, we further observe that, although at the location of the brane we have a physically acceptable situation with $\rho>0$ and $\rho+p_{r}>0$, both components $\rho$ and $p_{r}$ change sign at a particular point along the extra dimension, thus leading to $y$ regimes where both the weak and strong energy conditions are violated. Therefore, the result drawn in the previous section that this energy distribution cannot be realized by a conventional form of energy comes as no surprise.

It is nevertheless important to check the behavior of any 


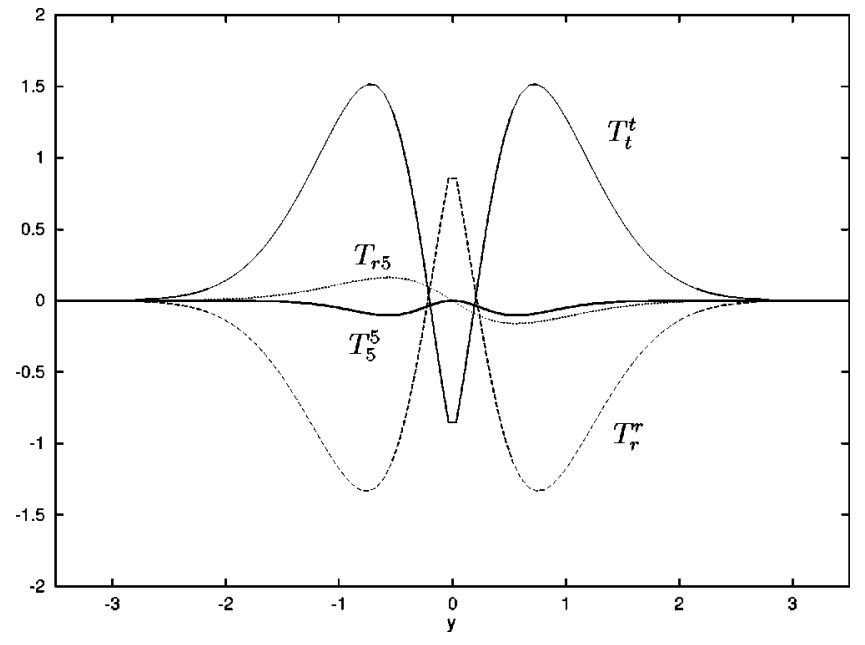

FIG. 2. The profile of the bulk energy-momentum tensor components, in terms of only the $y$ coordinate and for fixed $r=2 r_{h}$, is shown for $\lambda=a=r_{h}=1$ and $w(y)=r_{h} e^{-a y^{2}}$. All components remain localized near the brane, creating a symmetric "shell."

curvature invariant quantity that can be constructed in terms of the metric ansatz (6.1). The "decaying" value of the black hole horizon, as one moves away from the brane, is ensured; however, one needs also to investigate whether the black hole singularity is also "localized" near the brane. For this reason, we construct the $R_{M N R S} R^{M N R S}$ curvature invariant, which, for the RS choice of warp factor, is given by the following expression:

$$
\begin{aligned}
R_{M N R S} R^{M N R S}= & 40 \lambda^{4}+\frac{12 w^{2} e^{4 \lambda|y|}}{r^{6}} \\
& +8 A^{\prime} w^{\prime}\left[\frac{w^{\prime 2}}{(r-w)^{3}}+\frac{w^{\prime \prime}}{(r-w)^{2}}\right] \\
& +\frac{2\left(w^{\prime \prime 2}+7 \lambda^{2} w^{\prime 2}\right)}{(r-w)^{2}} \\
& +\frac{w^{\prime 2}}{(r-w)^{3}}\left[4 w^{\prime \prime}+\frac{11 w^{\prime 2}}{4(r-w)}\right] \\
& +\frac{2 w^{\prime 2}(3 r-2 w) e^{2 \lambda|y|}}{r^{3}(r-w)^{2}}
\end{aligned}
$$

The first term in the above expression is due to the presence of the bulk cosmological constant and is present also in the case of a $y$-independent four-dimensional metric tensor. The second term is the one that controls the behavior of the black hole singularity in the case of the RS-Schwarzschild solution, Eq. (1.1). We observe that the profile of this term along the extra dimension, in this particular case, is radically different. The presence of the additional scaling factor $e^{-2 a y^{2}}$, which comes from the expression of the "decaying" black hole horizon, causes this term to vanish at a large distance from the brane. In this way, the singularity at the AdS hori- zon disappears and, moreover, the black hole singularity itself "decays" as one moves away from the brane, thus leading to the localization of the black hole. However, there appear additional terms, which owe their existence to the $y$ dependence of the four-dimensional metric tensor. These terms give rise to a new singularity in the five-dimensional spacetime, which is located near the black hole horizon and dies out as one moves away from the brane. It can be easily shown that this new singularity is characteristic only of the particular five-dimensional spacetime which is described by the metric tensor, Eq. (6.1). Clearly, there are more that one five-dimensional spacetimes, each one possibly with a different global structure, that reduce to the same black hole solution on the brane. An alternative choice of the fivedimensional spacetime, which also induces a Schwarzschild solution on the brane but without possessing a horizon, might not have this problem. As such, we consider the following ansatz:

$$
\begin{aligned}
d s^{2}= & e^{2 A(y)}\left\{\frac{32 M(y)^{3} e^{-r / 2 M(y)}}{r}\left(-d v^{2}+d u^{2}\right)\right. \\
& \left.+r^{2}\left(d \theta^{2}+\sin ^{2} \theta d \varphi^{2}\right)\right\}+d y^{2},
\end{aligned}
$$

which is a five-dimensional analogue of the KruskalSzekeres black hole solution, $r$ being a function of $(v, u, y)$. Note that, on the brane, both Ansätze, (6.1) and (6.10), reduce to the same black hole solution: the two fourdimensional line elements are related by a coordinate transformation, $\quad(t, r) \leftrightarrow(v, u)$, and describe the same 4D spacetime. However, the embedding of these line elements in an extra dimension breaks their equivalence, a fact which is reflected in the different sets of curvature invariant quantities determined for the 5D line elements (6.1) and (6.10). The above metric ansatz is not plagued by the presence of a horizon and, in turn, no new singularity appears either in the expression of the scalar curvature or in the components of the bulk energy-momentum tensor. Unfortunately, for this alternative choice, a decaying value of the black hole horizon fails to either eliminate the singularity at the AdS horizon or to localize the black hole singularity. We might therefore conclude that the optimum choice of the five-dimensional metric tensor, which would be capable of sustaining localized black holes without the appearance of new singularities, is, in principle, a possible but rather difficult task.

Let us note, at this point, that the distribution of bulk matter around the brane and the black hole horizon should not be allowed to destroy the assumed staticity of the solution by generating an effective cosmological constant on the brane. Going back to the action (2.1) of our five-dimensional gravitational theory, substituting the $5 \mathrm{D}$ scalar curvature in terms of the metric tensor (6.1) and integrating over the extra dimension, we can easily evaluate the expression for the four-dimensional effective cosmological constant to be 


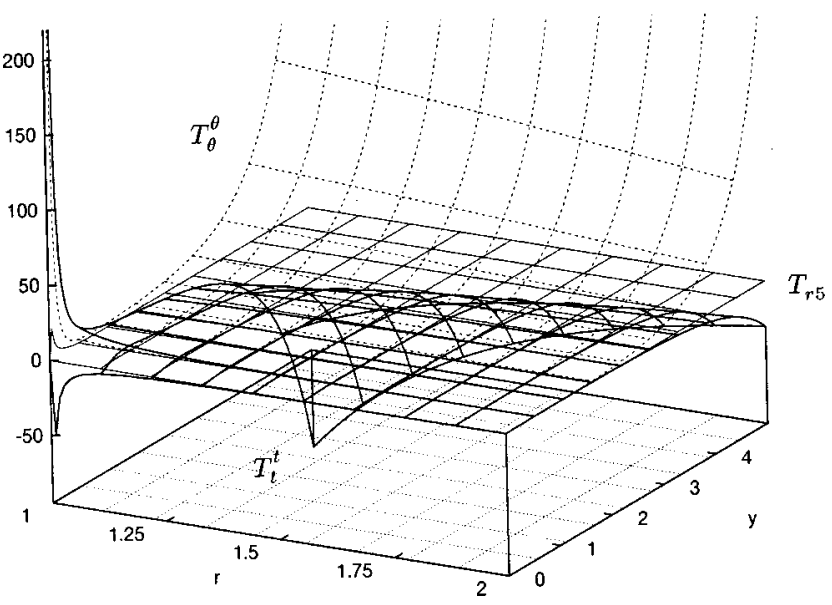

FIG. 3. The plot depicts the profile of the $T_{t}^{t}, T_{\theta}^{\theta}$ and $T_{r 5}$ components of the bulk energy-momentum tensor for the metric ansatz (6.12) and $\lambda=a=r_{h}=1$. All components (apart from $T_{r 5}$ ) diverge at $y$-infinity, violating any notion of localization of matter around the brane.

$$
\Lambda=\frac{1}{3}\left(\sigma-\frac{6 \lambda}{\kappa^{2}}\right)+\int d y e^{-4 \lambda|y|}\left[\frac{w^{\prime 2}}{4 \kappa^{2}(r-w)^{2}}+\mathcal{L}_{B}\right] .
$$

The first term in the above expression vanishes identically because of the fine-tuning between the bulk cosmological constant and the brane tension, which is valid in the original RS model and holds also in our case. The second term comes from the extra $y$ dependence of the metric due to the function $w(y)$ and the assumed bulk energy distribution that supports this extra dependence. If we want $\Lambda$ to be zero, then, the second term should also vanish, thus leading to a constraint on the bulk Lagrangian. Note that, under this constraint, no trace of the bulk matter distribution is left in the fourdimensional effective theory and, therefore, any particular features of $T_{N}^{M}$ cannot possibly affect the four-dimensional system.

As shown above, the ansatz for $U(r, y)$ used in Eq. (6.1) is not the only choice that fulfills our constraints for the four-dimensional metric function. Moreover, the ansatz (4.9) used in the previous sections is not the most general one, since the $(r, y)$-dependent part of $U(r, y)$ need not be in a factorized form. An alternative metric ansatz that also respects our demands for the behavior of the 4D metric function near as well as far away from the brane is

$$
\begin{aligned}
d s^{2}= & e^{2 A(y)}\left\{-\left(1-\frac{r_{h}}{\sqrt{r^{2}+y^{2}}}\right) d t^{2}\right. \\
& +\left(1-\frac{r_{h}}{\sqrt{r^{2}+y^{2}}}\right)^{-1} d r^{2} \\
& \left.+r^{2}\left(d \theta^{2}+\sin ^{2} \theta d \varphi^{2}\right)\right\}+d y^{2} .
\end{aligned}
$$

The above ansatz also reduces to the Schwarzschild solution in the limit $y \rightarrow 0$, recovering the black hole metric function on the brane. In the other asymptotic regime, $y \rightarrow \pm \infty$, the term proportional to $r_{h}$ shrinks to zero, as demanded, for any value of $r$. Following the same method as for the previous metric ansatz (6.1) and isolating those terms in Einstein's equations whose presence is attributed to the bulk matter distribution, we determine the components of $T_{N}^{M}$ whose behavior with respect to the $(r, y)$ coordinates is shown in Fig. 3 . From the figure it becomes evident that the particular matter distribution is highly unphysical: although the behavior of all components near the brane is identical to the one of the previous case, the situation changes radically at the other asymptotic regime of the extra dimension. All components, apart from the off-diagonal one, $T_{r 5}$, that tends to zero, start to diverge rapidly, eventually acquiring infinite values near the AdS horizon. This behavior obviously violates any desired notion of localization of matter around the brane, as it is physically unreasonable to accept a diverging distribution of matter at infinity in order to support a localized black hole solution near the brane. We therefore conclude that a welldefined behavior of the metric function, near or away from the brane, does not guarantee the same behavior for the distribution of bulk matter.

\section{CONCLUSIONS}

In this paper, we have addressed the issue of the localization of brane-world black holes along the extra dimension by investigating the existence of $y$-dependent four-dimensional line elements that would allow for an extra scalingdecaying — of the physical value of the black hole horizon in terms of the extra coordinate, while reducing to a conventional black hole solution on the brane.

Throughout the paper, a spherically symmetric, nonfactorizable $y$-dependent ansatz for the four-dimensional metric tensor was made, i.e., $g_{\mu \nu}=e^{2 A(y)} \hat{g}_{\mu \nu}(x, y)$, with the assumption that, in the limit $y \rightarrow 0$, our ansatz should reduce to a well-known four-dimensional black hole solution. Apart from the desired behavior near as well as away from the brane, our ansatz was chosen to satisfy the jump conditions with a continuous $\hat{g}_{\mu \nu}(x, y)$, while the discontinuity at the brane was taken care of by the warp factor. We restricted our investigation by considering the case $\hat{g}_{t t}=-\hat{g}_{r r}^{-1}$ and taking the warp factor to correspond either to the simple RandallSundrum choice $A(y)=-\lambda|y|$ or to the one of the RSAdS/dS solutions. We believe that the essential features of this analysis will remain true, even in the case that we generalize our ansatz and move beyond these choices.

In the first part of the paper, we considered the cases of empty bulk and bulk containing either a scalar or a gauge field that might support such $y$-dependent solutions. In particular cases, the most general form of the metric function $U(r, y)$ compatible with the equations of motion was derived by directly integrating the off-diagonal component of Einstein's equations. In more complicated cases, a particular ansatz based on our assumptions and physically motivated by the form of the previously derived solutions was used. In all 
cases considered, of an empty, scalar-field- or gauge-fielddominated bulk, no solutions supporting the desired $y$ dependence of the 4D metric function and, thus, the scaling of the black hole horizon with respect to the extra dimension, independently of the one of the warp factor, were derived. The assumed $y$ dependence was inconsistent with either the continuity of the 4D metric function $U(r, y)$ across the brane or the reality of the same function, and in all cases the $y$-independent Schwarzschild or AdS/dS-Schwarzschild solutions emerged as the unique solutions of the system.

In the second part of the paper, we followed an alternative approach, in which a particular ansatz that supports the scaling of the black hole horizon with the extra dimension was given as input and the nature of the corresponding bulk energy-momentum tensor was to be determined. The ansatz described a $y$-dependent generalization of the Schwarzschild solution with a Randall-Sundrum type of warp factor. The value of the black hole horizon scaled as $r_{h}(y)=r_{h} e^{-a y^{2}}$, which ensured the "decaying," and eventually the vanishing, of the black hole horizon as well as the continuity of the 4D metric function. Knowing the exact form of the fivedimensional line element, we were able to solve for the components of the corresponding bulk energy-momentum tensor and to check their behavior, with respect to the brane $(r)$ and bulk $(y)$ coordinates. According to our analysis, for each AdS slice $\left(y=y_{*}\right)$, the components of $T_{N}^{M}$ closely follow the behavior of the metric tensor by diverging near the black hole horizon and vanishing asymptotically $[$ as $\mathcal{O}(1 / r)]$ away from it. Regarding their $y$ profile, all components turned out to respect the notion of localization of matter around the brane: the matter distribution has its peak slightly off the brane and rapidly decreases as we move away from the brane. Both the bulk energy-momentum tensor and the black hole horizon vanish well before the AdS horizon is reached. The energy density and radial pressure of the bulk matter are shown to satisfy a stiff equation of state throughout the extra spacetime while the change of their sign in particular $y$ regimes confirms the non-conventional type of nature of the bulk matter. The calculation of curvature invariant quantities, such as the square of the Riemann tensor, reveals the disappearance of the singularity at the AdS horizon and the localization of the black hole singularity around the brane. However, a second, although localized, singularity appears at the black hole horizon due to the singular behavior of the bulk energymomentum tensor at this point. By considering an alternative choice for the five-dimensional metric tensor, which describes a spacetime with a distinctly different topology from the first one but which also reduces to the same black hole solution on the brane, we were able to demonstrate that this singularity is merely due to the particular choice of the fivedimensional spacetime. Any additional choices, however, considered for the five-dimensional spacetime, that were free from the second singularity at the induced black hole horizon, were instead plagued by the singularity at the AdS horizon. It would, therefore, be interesting to investigate whether this bulk singularity, located either at the AdS horizon or at a finite distance from the brane, is a generic feature of every five-dimensional spacetime that induces a black hole solution on the brane (as it is, for example, argued in the case of a radiating brane on the grounds of the AdS/CFT correspondence [23]) or whether an alternative, well-defined $5 \mathrm{D}$ spacetime indeed exists. We leave this investigation for a future work.

As a concluding remark, let us note that our analysis cannot be considered as a no-go theorem analysis for the existence of realistic localized black holes by means of the decaying of their horizon value with the extra dimension. The analysis in Secs. III, IV and V does not cover every possible choice for the metric function $U(r, y)$. However, a general analysis along the same lines, which would be able to exclude any form of $y$-dependent metric function, is extremely difficult. In the same way, the ansatz considered in Sec. VI is only one of the many Ansätze that might satisfy our assumptions. Our analysis clearly suggests that the localization of brane-world black holes strongly depends on the nature of the bulk matter distribution. The localized black hole solutions, of the type considered in this paper, were shown to demand an exotic form of bulk matter. The quest for localized black hole solutions, supported by a matter distribution that has a physically acceptable interpretation, is to be continued.

\section{ACKNOWLEDGMENTS}

P.K. is deeply grateful to John March-Russell and Valery Rubakov for useful discussions. K.T. is thankful to A. Kehagias for a number of illuminating discussions. P.K. and K.T. acknowledge the financial support of the EU RTN contract No. HPRN-CT-2000-00148 and the EU RTN contract No. HPRN-CT-2000-00152.
[1] N. Arkani-Hamed, S. Dimopoulos, and G. Dvali, Phys. Lett. B 429, 263 (1998); Phys. Rev. D 59, 086004 (1999).

[2] I. Antoniadis, N. Arkani-Hamed, S. Dimopoulos, and G. Dvali, Phys. Lett. B 436, 257 (1998).

[3] L. Randall and R. Sundrum, Phys. Rev. Lett. 83, 3370 (1999).

[4] L. Randall and R. Sundrum, Phys. Rev. Lett. 83, 4690 (1999).

[5] J. Lykken and L.J. Randall, J. High Energy Phys. 06, 014 (2000); K. Skenderis and P.K. Townsend, Phys. Lett. B 468, 46 (1999); K. Behrndt and M. Cvetic, ibid. 475, 253 (2000); A. Chamblin and G.W. Gibbons, Phys. Rev. Lett. 84, 1090
(2000); O. DeWolfe, D.Z. Freedman, S.S. Gubser, and A. Karch, Phys. Rev. D 62, 046008 (2000); C. Csaki, J. Erlich, T.J. Hollowood, and Y. Shirman, Nucl. Phys. B581, 309 (2000); S.B. Giddings, E. Katz, and L.J. Randall, J. High Energy Phys. 03, 023 (2000); H. Kudoh and T. Tanaka, Phys. Rev. D 64, 084022 (2001).

[6] A. Chamblin, S.W. Hawking, and H.S. Reall, Phys. Rev. D 61, 065007 (2000).

[7] I. Giannakis and H.c. Ren, Phys. Rev. D 63, 024001 (2001).

[8] R. Gregory and R. Laflamme, Phys. Rev. Lett. 70, 2837 
(1993).

[9] R. Emparan, G.T. Horowitz, and R.C. Myers, J. High Energy Phys. 01, 007 (2000); J. Garriga and M. Sasaki, Phys. Rev. D 62, 043523 (2000); A. Chamblin, C. Csaki, J. Erlich, and T.J. Hollowood, ibid. 62, 044012 (2000); N. Dadhich, R. Maartens, P. Papadopoulos, and V. Rezania, Phys. Lett. B 487, 1 (2000); S. Nojiri, O. Obregon, S.D. Odintsov, and S. Ogushi, Phys. Rev. D 62, 064017 (2000); A. Chamblin, H.S. Reall, H.a. Shinkai, and T. Shiromizu, ibid. 63, 064015 (2001); M. Rogatko, Phys. Rev. D 64, 064014 (2001); I. Oda, hep-th/0008055; M. Bruni, C. Germani, and R. Maartens, Phys. Rev. Lett. 87, 231302 (2001); S.I. Vacaru and E. Gaburov, hep-th/0108065; A.J. Medved, Class. Quantum Grav. 19, 405 (2002).

[10] C. Gomez, B. Janssen, and P.J. Silva, J. High Energy Phys. 04, 027 (2000); A. Kamenshchik, U. Moschella, and V. Pasquier, Phys. Lett. B 487, 7 (2000); D. Youm, ibid. 515, 170 (2001); C. Grojean, F. Quevedo, G. Tasinato, and I. Zavala C., J. High Energy Phys. 08, 005 (2001); D. Youm, Mod. Phys. Lett. A 16, 1703 (2001); S. Nojiri, S.D. Odintsov, and S. Ogushi, Int. J. Mod. Phys. A 16, 5085 (2001); R.G. Cai and Y.Z. Zhang, Phys. Rev. D 64, 104015 (2001); D. Birmingham and M. Rinaldi, Mod. Phys. Lett. A 16, 1887 (2001).

[11] H. Lu and C.N. Pope, Nucl. Phys. B598, 492 (2001); C.V. Johnson and R.C. Myers, Phys. Rev. D 64, 106002 (2001); S.S. Gubser, A.A. Tseytlin, and M.S. Volkov, J. High Energy Phys. 09, 017 (2001).

[12] H.S. Reall, Phys. Rev. D 64, 044005 (2001); G.T. Horowitz and K. Maeda, Phys. Rev. Lett. 87, 131301 (2001); J.P. Gregory and S.F. Ross, Phys. Rev. D 64, 124006 (2001); S. Yoon, hep-th/0106286.

[13] M.R. Mbonye, Phys. Rev. D 60, 124007 (1999); S.W. Hawking and H.S. Reall, ibid. 61, 024014 (2000); P. Kraus, J. High
Energy Phys. 12, 011 (1999); A.M. Awad and C.V. Johnson, Phys. Rev. D 63, 124023 (2001); C. Cadeau and E. Woolgar, Class. Quantum Grav. 18, 527 (2001); M.S. Modgil, S. Panda, and G. Sengupta, hep-th/0104122; S.I. Vacaru, hep-th/0110250; hep-th/0110284; R. Emparan and H.S. Reall, hep-th/0110260; U.H. Danielsson, hep-th/0110265.

[14] P.C. Argyres, S. Dimopoulos, and J. March-Russell, Phys. Lett. B 441, 96 (1998); R. Emparan, G.T. Horowitz, and R.C. Myers, Phys. Rev. Lett. 85, 499 (2000); R. Casadio and B. Harms, Phys. Lett. B 487, 209 (2000); Phys. Rev. D 64, 024016 (2001); F.C. Adams, G.L. Kane, M. Mbonye, and M.J. Perry, Int. J. Mod. Phys. A 16, 2399 (2001); S. Dimopoulos and G. Landsberg, Phys. Rev. Lett. 87, 161602 (2001); M.B. Voloshin, Phys. Lett. B 518, 137 (2001); K. Cheung, hep-ph/0110163.

[15] N. Kaloper, Phys. Rev. D 60, 123506 (1999).

[16] H.B. Kim and H.D. Kim, Phys. Rev. D 61, 064003 (2000).

[17] P. Kanti, I.I. Kogan, K.A. Olive, and M. Pospelov, Phys. Lett. B 468, 31 (1999); Phys. Rev. D 61, 106004 (2000).

[18] A. Karch and L. Randall, J. High Energy Phys. 05, 008 (2001).

[19] T. Hirayama and G. Kang, Phys. Rev. D 64, 064010 (2001).

[20] I. Giannakis and H.c. Ren, Phys. Rev. D 63, 125017 (2001); 64, 065015 (2001).

[21] M. Visser, Phys. Lett. B159, 22 (1985).

[22] B. Carter, in Black Holes, Proceedings of the 1972 Les Houches Summer School of Theoretical Physics, edited by C. DeWitt and B.S. DeWitt (Gordon and Breach, New York, 1973).

[23] S.S. Gubser, Phys. Rev. D 63, 084017 (2001); S. Nojiri and S.D. Odintsov, J. High Energy Phys. 07, 049 (2000); L. Anchordoqui, C. Nunez, and K. Olsen, ibid. 10, 050 (2000); I. Savonije and E. Verlinde, Phys. Lett. B 507, 305 (2001); A. Hebecker and J. March-Russell, Nucl. Phys. B608, 375 (2001). 